\title{
ISS OF POLARIZED COMPACT EXTRAGALACTIC RADIO SOURCES
}

\author{
BARNEY RICKETT \\ University of California, San Diego, U.S.A.
}

\begin{abstract}
It is shown that the detailed inter-relations of the $8.6 \mathrm{GHz}$ IDV in Stokes' parameters $I, Q$ and $U$ of the quasar $0405-385$, as quantified by their auto and cross correlations, can be successfully modelled by interstellar scintillation (ISS) of a double source with two components separated by about 10 micro-arcseconds. Though this is not a unique source model it confirms that ISS can explain quantitatively the rapid variations in both flux and linear polarization. Thus there is no evidence in support of an intrinsic component in the IDV.
\end{abstract}

Keywords: Interstellar, scintillation, AGN, polarization

\section{Intra-Day Variations of AGNs}

Intra-day variation (IDV) in cm-wavelength flux density of a few Active Galactic Nuclei (AGNs) has been recognised for over ten years (Quirrenbach et al., 1989a). As for low frequency variations (over much longer times), the classic time scale argument and an assumption that the variation is intrinsic lead to apparent brightness temperatures that are 3-6 orders of magnitude above the inverse Compton limit. With the discovery of two even more rapidly varying sources (KedzioraChudczer et al., 1997) and (Dennett-Thorpe and de Bruyn, 2000), the intrinsic interpretations are stretched another three orders of magnitude in apparent brightness. Alternatively IDV could be due to interstellar scintillations (ISS), which was proposed in the latter two works and was also advanced as the explanation of the archetypical IDV source 0917+624 (Rickett, 1990 and Rickett et al., 1995). In the latter work the authors found ISS to explain many detailed features of the variations over a wide range of frequencies; they assumed the source at $\mathrm{cm}$ wavelengths to be dominated by a compact component with a brightness temperature of $6 \times 10^{12} \mathrm{~K}$, whose diameter increased linearly with wavelength, as might be expected for an inhomogeneous synchrotron emission process with flux limited by self-absorption at each wavelength.

Many of the IDV sources also show significant levels of rapidly varying linear polarization, often more rapid than the total flux (Quirrenbach et al., 1989b). While intrinsic models have been proposed for such variations, Rickett et al. (1995) argued that the polarization changes could also be explained as ISS. The goal here is to present an ISS interpretation of the rapid variations in linearly polarized flux 
for the quasar 0405-385, during its episode of very rapid IDV as observed with the Australia Telescope by Kedziora-Chudczer et al. (1997).

\section{ISS in Stokes Parameters}

I outline here a theory for the ISS in the Stokes' parameters for a source whose structure in $Q$ and $U$ may differ from that in $I$. Parallel equations exist for $V$. ISS fluctuations in $I$ will be accompanied by fluctuations in the linear polarization parameters $Q$ and $U$ (used here because they sum linearly from different regions of emission). Observationally, one can quantify the relationship between fluctuations in $I$ and $Q$, say, by their auto- and cross-correlation functions. I now outline the theory for these correlations, assuming weak or refractive ISS and a screen geometry; results for an extended scattering medium will be given in a more complete paper, in preparation.

Consider a scattering layer (screen) of thickness $\delta z$ at distance $z$ from an observer who receives polarized waves from a source, whose brightness distribution in $I$ is $B_{I}(\vec{\theta})$. Let $\Delta I_{o}(\vec{s})$ be the pattern of intensity fluctuation about its mean at transverse location $\vec{s}$ for a unit flux point-source located on the $z$-axis. The net intensity is the sum of shifted terms weighted by each brightness element:

$$
\Delta I(\vec{s})=\iint \Delta I_{o}(\vec{s}-z \vec{\theta}) B_{I}(\vec{\theta}) d^{2} \vec{\theta}
$$

where the angles are all small and infinite limits are implied in this and subsequent integrals. Exactly similar equations can be written for $\Delta Q$ and $\Delta U$ for a source with polarized brightness functions $B_{Q}(\vec{\theta})$ and $B_{U}(\vec{\theta})$, since the ISS in, say, $Q$ due to each source element is given simply by $\Delta I_{o}(\vec{s}-z \vec{\theta}) B_{Q}(\vec{\theta}) d^{2} \vec{\theta}$.

The important underlying assumption here is that the scintillation conditions in the two modes of propagation through the magneto-active plasma are essentially identical. For radio-astronomical signals travelling through the ISM the right and left circular modes have a phase difference governed by the rotation measure on that line-of-sight for which a typical value might be $30 \lambda^{2}$ radians with wavelength $\lambda$ in meters. This is very small compared to the total phase increment due to the plasma, for which a typical value might be $3 \times 10^{8} \lambda$ radians. ISS is governed by the field coherence scale $s_{d}$, over which this total plasma phase changes by one radian rms, in comparison to the Fresnel scale $r_{f}=\sqrt{2 \lambda / 2 \pi}$. If $s_{d}>r_{f}$ we have weak ISS (rms flux $<$ mean flux) and vice-versa for strong ISS. The figures given ensure that over the Fresnel scale for weak ISS the phase difference between the two modes is only about a millionth of a radian. Then the scintillation patterns in $Q, U$ and $V$ for an isolated point source are identical to those in $I$ except for constants that represent the (steady) source polarization. 
For a point source, ISS caused by a screen is statistically homogeneous and has a spatial auto-correlation which depends only on the spatial separation $\vec{\sigma}$, not on absolute position $\vec{s}$ :

$$
C_{o o}(\vec{\sigma})=<\Delta I_{o}(\vec{s}) \Delta I_{o}(\vec{s}+\vec{\sigma})>.
$$

Cross-correlations versus spatial offsets can be defined for each pair of observed Stokes' parameters; e.g. for $I$ and $Q$ :

$$
C_{I Q}(\vec{\sigma})=<\Delta I(\vec{s}) \Delta Q(\vec{s}+\vec{\sigma})>.
$$

Substituting Equation (1) and the parallel equation for $\Delta Q$ one obtains:

$$
C_{I Q}(\vec{\sigma})=\iint C_{o o}(\vec{\sigma}-z \vec{\alpha}) R_{I Q}(\vec{\alpha}) d^{2} \vec{\alpha}
$$

where

$$
R_{I Q}(\vec{\alpha})=\iint B_{I}(\vec{\beta}-\vec{\alpha} / 2) B_{Q}(\vec{\beta}+\vec{\alpha} / 2) d^{2} \vec{\beta} .
$$

A version of these equations with subscripts $I I$ in place of $I Q$ given $C_{I I}$ in terms of $R_{I I}$ (Little and Hewish, 1966); evidently there are similar results for the other auto and cross correlations $(Q Q, U U, I U, Q U)$.

At present no extragalactic radio source has a small enough angular size to exhibit diffractive ISS. Consequently, we are concerned with refractive ISS in strong scintillation of with weak ISS. In both cases expressions for the point source correlation (2) are most simply given in the wavenumber domain as a cross-spectrum, as in Appendix B of Rickett et al. (1995). Analogous equations give the crossspectrum for ISS in $I$ and $Q$, which is the Fourier transform of $C_{I Q}(\vec{\sigma})$. Equations like (4) give each of the six possible correlation functions as convolutions of $C_{o o}(\vec{\sigma})$ with various auto and cross correlations of the corresponding brightness functions.

In isotropic weak scintillation the form of $C_{o o}(\vec{\sigma})$ is a smooth circular function of width $r_{f}$ peaking at the origin. When the fine structure of the plasma is anisotropic, this 'point spread function' becomes anisotropic. An example is shown in Figure 1. The key point here is that the function has pronounced negative 'over-shoot' when the velocity vector is aligned approximately perpendicular to the filamentary plasma structures. This feature is important since it matches the 0405385 observations at 4.8 and $8.6 \mathrm{GHz}$, which have a quasi-oscillatory appearance with a $50 \%$ negative overshoot in their autocorrelations and so require an axial ratio less than 0.3 as shown in Figure 2.

Figure 2 shows a comparison of the correlations in $I, Q$ and $U$ at $8.6 \mathrm{GHz}$ with weak ISS theory. The theoretical equations, in terms of spatial offsets, are used to model the six temporal auto and cross correlations (at time lag $\tau$ ) by setting $\vec{\sigma}=\vec{V}_{\text {iss }} \tau$, for the motion of the observer through a time stationary scintillation pattern at velocity $\vec{V}_{\mathrm{iss}}$. Of course we do not know the orientation of this velocity 


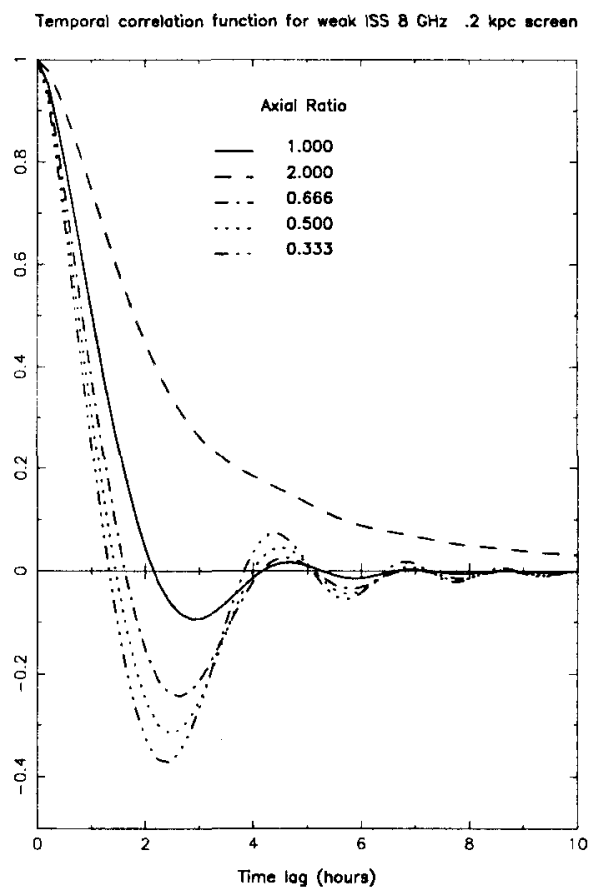

Figure 1. Weak ISS for anisotropic scattering at $8 \mathrm{GHz}$ from a Kolmogorov sereen at a distance of $200 \mathrm{pc}$; slices (at $50 \mathrm{~km} \mathrm{~s}^{-1}$ ) along the axis of $C_{O O}(\vec{\sigma})$, where axial ratio $<1$ corresponds to velocity vector perpendicular to the plasma filaments.

vector relative to the axis of the anisotropy, but for a successful ISS explanation of IDV we require that the velocity should not be aligned along the filamentary axis (axial ratios $>1$ ). Calculations like those in Figure 1 show that the negative overshoot is visible over a wide range of orientations and only disappears when the velocity and the filament axes are aligned to within a radian angle about equal to the axial ratio $(<1)$. Here this is less than about 0.3 radians, giving a strong statistical preference for orientations that would show negative overshoots.

The particular model shown here is scintillation in a screen at $38 \mathrm{pc}$ from the Earth with a Kolmogorov phase spectrum and a source model consisting of two Gaussian components with nearly orthogonal linear polarizations separated by $10 \mu$ as. $70 \%$ of the flux is in one component with $5 \mu$ as diameter and $2 \%$ linear polarization at angle 3 degrees; the other component has $30 \%$ of the flux with same diameter and $5 \%$ linear polarization at angle -85 degrees. These parameters have been optimized to fit the observed correlations, and the success in fitting is sufficient to argue that ISS can indeed explain the IDV in $I, Q$ and $U$. However, the solution does not yet include a calculated scintillation index and an inferred scattering measure for the screen, nor does it model how the components depend 
Weak ISS $8.6 \mathrm{GHz}$, Screen ot $38 . \mathrm{pc}$
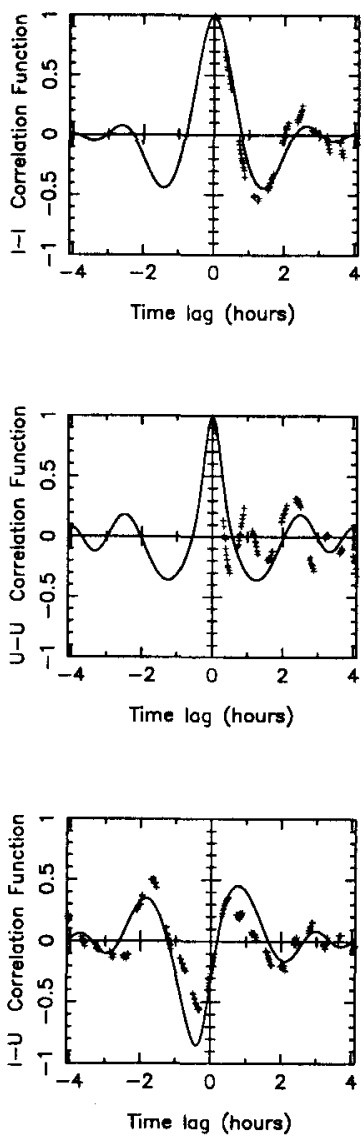

Weck ISS $8.6 \mathrm{GHz}$, Screen at 38 . pc
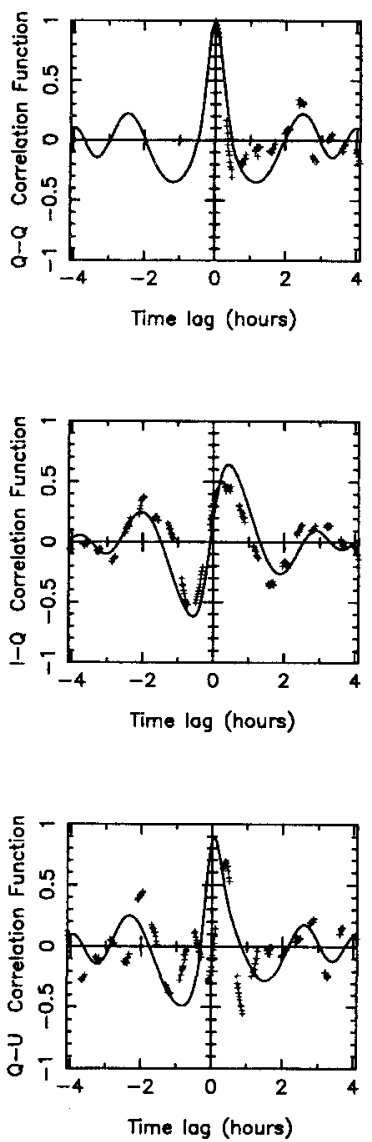

Figure 2. Correlation analysis for IDV in the Stokes' parameters from quasar 0405-385 at 8640 MHz. Pulses are observations from the Australia Telescope compact array in June 8,9,10 1996. Solid line is a specific ISS model, as described in the text.

on frequency. The goal is a fully self-consistent model for the $I, Q$ and $U$ at 8640 and $4800 \mathrm{MHz}$, for which the IDV are highly correlated.

In the model fitting the velocity was $36 \mathrm{~km} \mathrm{~s}^{-1}$ which is the projected perpendicular velocity of the Earth relative to the local standard of rest, which has been shown to be a reasonable estimate of the rest frame of the scattering plasma (Rickett, Coles and Markkanen, 2000). With the velocity assumed known, the distance to the screen and the source structure combine to determine the time-scale of the ISS. In fitting the shapes of the correlations one cannot uniquely determine both. Thus there is a family of similar models with the product of screen distance and source angular scale approximately constant. Moving the screen to a greater distance requires proportionately smaller angular sizes. However, from analyzing the 
intensity distribution function we can infer that the compact source has a maximum scintillation index of 0.4 at $4.8 \mathrm{GHz}$, which requires that the source structure is large enough to partially suppress the ISS. This puts a lower limit on the source size and an upper limit on the screen distance. A preliminary estimate gives a maximum screen distance of $100 \mathrm{pc}$, but, with the double source model needed to model the polarization, this constraint will require numerical solution over the family of models.

\section{Acknowledgements}

I thank D. Jauncey and L. Kedziora-Chudczer for generously providing their polarization data; a full paper co-authored with them is in preparation; I thank M. Walker for valuable discussions.

\section{References}

Dennett-Thorpe, J. and de Bruyn, A.G.: 2000, The Discovery of a Microarcsecond Quasar: J1819+3845, Astrophys. J. 529, L65-L68.

Kedziora-Chudczer, L., Jauncey, D.L., Wieringa, M.H., Walker, M.A., Nicholson, G.D., Reynolds, J.E. and Tzioumis, A.K.: 1997, PKS 0405-385: The Smallest Quasar?, Astrophys. J. 490, L9-12.

Little, L.T. and Hewish, A.: 1966, Interplanetary Scintillation and its Relation to the Angular Structure of Radio Sources, Mon. Not. R. Astron. Soc. 134, 221-237.

Quirrenbach, A., Witzel, A., Krichbaum, T.P., Hummel, C.A., Alberdi, A. and Schalinski, C.: 1989a, Rapid Variability of Extragalactic Radio Sources, Nature 337, 442-444.

Quirrenbach, A., Witzel, A., Qian, S.J., Krichbaum, T.P., Hummel, C.A. and Alberdi, A.: 1989b, Rapid radio polarization variability in the quasar 0917+624, Astron. Astrophys. 226, L1-L4.

Rickett, B.J.: 1990, Radio propagation through the Turbulent Interstellar Plasma, Annu. Rev. Astron. Astrophys. 28, 561-605.

Rickett, B.J., Coles, W.A. and Markkanen, J.: 2000, Interstellar Scintillation of Pulsar B0809+74, Astrophys. J. 533, 304-319.

Rickett, B.J., Quirrenbach, A., Wegner, R., Krichbaum, T. and Witzel, A.: 1995, Interstellar Scintillation of the Radi Source 0917+624, Astron. Astrophys. 293, 479-492. 